\title{
Spububaúde
}

\section{O impacto do Omega-3 no tratamento da leucemia em crianças e adolescentes}

\author{
The impact of Omega- 3 on the treatment of leukemia in children and adolescents
}

\author{
Jennyfhe Emannuelly da Silva Pereira ${ }^{1}$, Maria Cecília da Silva $^{2}{ }^{\bullet}$, Camila Chiara Pereira de \\ Oliveira $^{30}$
}

\begin{abstract}
${ }^{1}$ Graduada em Nutrição no Centro Universitário UNIFAVIP/ Wyden, Caruaru, Pernambuco, Brasil. ${ }^{2}$ Graduada em Nutrição no Centro Universitário UNIFAVIP/ Wyden, Caruaru, Pernambuco, Brasil. ${ }^{3}$ Docente do curso de Nutrição no Centro Universitário UNIFAVIP/ Wyden, Caruaru, Pernambuco, Brasil. *Autor para correspondência. E-mail: jennyfhe@hotmail.com
\end{abstract}

\begin{abstract}
Resumo: Há diversos tipos de neoplasias, sendo as leucemias os tipos que mais atingem crianças e adolescentes. Afetando a medula óssea que é a responsável pela fabricação dos formantes do sangue. No Brasil, o câncer representa a primeira causa de morte entre crianças e adolescentes de 01 a 19 anos. A presente pesquisa trata-se de um estudo de revisão da literatura e teve como objetivo descrever o impacto do omega-3 no tratamento da leucemia em crianças e adolescentes. Foi realizada uma revisão de literatura, nas bases de dados eletrônicas PubMed e Scielo com artigos publicados entre 2009 a 2019. Estudos evidenciam a importância do tratamento nutricional com imunomoduladores para os pacientes com neoplasias, principalmente o uso de ômega-3 como estratégia na melhora do quadro clínico. Os resultados do estudo mostraram evidências que sugere benefícios consideráveis à saúde, incluindo efeitos anti-inflamatórios e efeitos antioxidantes com a utilização dos ácidos graxos ômega-3 em pacientes com câncer.
\end{abstract}

Palavras-chaves: Crianças, Adolescentes, Leucemia mieloide aguda, Fatores imunológicos.

\begin{abstract}
There are several types of neoplasms, and leukemias are the types that most affect children and adolescents. Affecting the bone marrow which is responsible for manufacturing blood formants. In Brazil, cancer represents the leading cause of death among children and adolescents aged between 1 and 19 years. This research is a literature review study and aimed to define the impact of omega- 3 in the treatment of leukemia in children and adolescents. A literature review was carried out in the electronic databases PubMed and Scielo with articles published between 2009 and 2019. Studies show the importance of nutritional treatment with immunomodulators for patients with cancer, especially the use of omega- 3 as a strategy to improve the clinical condition. The study results indistinctly that indistinctly considerable health benefits, including anti-inflammatory effects and antioxidant effects with the use of omega- 3 fatty acids in cancer patients.
\end{abstract}

Keywords: Children, Adolescents, Acute myeloid leukemia, Immunological factors.

\section{Introdução}

Há diversos tipos de neoplasias, sendo as leucemias os tipos que mais atingem crianças e adolescentes. Sabendo que a medula óssea, é a responsável pela fabricação dos formantes do sangue: glóbulos vermelhos, responsáveis pelo transporte de oxigênio de nosso organismo, glóbulos brancos, que atacam as infecções, e plaquetas, responsáveis pela coagulação do sangue, impedindo que ocorra hemorragias. A leucemia acontece quando os leucócitos, um tipo de glóbulo branco, perdem a função de defesa e passam a se desenvolver de maneira descontrolada. São diversas as linhagens celulares que se formam da medula óssea, por isso as leucemias estão dividias em dois grandes grupos: mieloide e linfoide. Além disso, também podem ser consideradas como agudas ou crônicas (ABRALE, 2016).

Como também nos países desenvolvidos, no Brasil, o câncer já está representa a primeira causa de morte entre crianças e adolescentes com faixa etária de 01 a 19 anos. Nas últimas décadas, houve um avanço no tratamento do câncer na infância e na adolescência, sendo esse profundamente significativo para a recuperação clínica do paciente. Nos dias atuais, cerca de $80 \%$ das crianças e adolescentes acometidos com a doença podem ser curados, se diagnosticados antecipadamente e tratados em centros especializados (INCA, 2018). 
Desta forma, a intervenção nutricional é de extrema importância no tratamento para esses pacientes tendo como ponto principal a execução de algumas metas necessária para a melhora e manutenção do indivíduo acometido com câncer, tais como: promover condições favoráveis para o paciente, minimizar os efeitos deletérios desta doença assim fornecendo uma melhor, condição de vida. Em relação aos nutrientes, atualmente, alguns estudos têm sugerido efeitos distintos desses nutrientes para esses pacientes, sobre o crescimento do tumor, incluindo a atividade antitumoral (Mahan et al., 2010; Perini et al., 2010; Mesquita et al., 2011; West et al., 2014; Garófolo et al.,2018).

Vale ressaltar, que a abordagem terapêutica e nutricional nos pacientes com neoplasias é fundamental para a melhora do quadro clínico do indivíduo acometido por essa enfermidade. Principalmente, quando se fala da utilização de ácidos graxos ômega-3. A utilização desse imunomodulador para pacientes com câncer, é um conceito novo e promissor, que vai além de seu potencial papel na imunidade, isto é, trabalha na reversão da caquexia do câncer, que promove a manutenção do peso e a melhora da massa muscular. A Combinação do tratamento tradicional associado à suplementação de ômega-3 parece ser uma estratégia eficaz em diferentes tipos de câncer (Elbarbary et al., 2015). Assim, esse estudo teve como objetivo descrever o impacto do omega3 no tratamento da leucemia em crianças e adolescentes.

\section{Revisão e discussão}

Este trabalho teve como finalidade a realização de uma revisão da literatura. Para essa revisão, buscaramse artigos nas bases de dados eletrônicas PubMed e Scielo publicados nas seguintes línguas, portuguesa e inglesa. Foram utilizados os descritores: Crianças “Children”, adolescentes "adolescents”, Leucemia mieloide aguda "Acute myeloid leukemia”, fatores imunológicos "immunological character”. Para a identificação e seleção dos estudos foram incluídos trabalhos que: abordassem o impacto do W3 no tratamento da leucemia em crianças e adolescentes, e publicados entre os anos de 2009 a 2019. Foram excluídos da pesquisa monografias e dissertações com o tema propostos. Foram utilizados 15 artigos nesta revisão.

Os estudos analisados na presente pesquisa demonstram que há benefícios no consumo de ômega-3 para pacientes com Leucemia. O Consenso Nacional em Nutrição Oncológica (INCA, 2011) descreve que dietas imunomoduladoras contendo ácidos graxos $\omega$ - 3 mostraram benefícios em pacientes com câncer.

A dieta imunomoduladora pode contribuir para a diminuição das complicações pós-cirúrgicas e para uma melhora na recuperação do peso, tendo grandes chances de influenciar significativamente na sobrevida do paciente. Assim, os ácidos graxos $\omega-3$ poderiam auxiliar no tratamento de neoplasias, atuando na produção de mediadores lipídicos com atividade inflamatória atenuada ou atuando na fase de resolução do processo inflamatório (Maruyama et al., 2010).

Vale mencionar, que o ômega-3 é fonte basicamente ácidos graxos insaturados, esse nutriente é encontrado em alimentos de origem vegetal e de origem animal. Sendo também um composto que apresenta as seguintes variações: como ALA presente na soja e canola, que são óleos vegetais. Bem como, EPA e DHA que são encontrados com maior frequência em peixes de águas frias e profundas, tendo como exemplo o salmão, sardinha, arengue e cavalinha. Dessa forma, esses alimentos podem compor o cardápio dos pacientes em tratamento com câncer com o intuito na melhora do quadro clínico desses indivíduos acometidos por essa enfermidade (Santos et al, 2013).

No estudo de Fahrmann e Hardman (2013) foram observadas células de linhagem leucêmicas onde sugerem que os lipídeos contendo ácidos graxos docosaexaenoico (DHA) e ecosapentaenóico (EPA), podem sensibilizar positivamente as células cancerígenas ou os tumores a fármacos antineoplásicos, e também preservar ou mesmo proteger os tecidos sadios, bem como, aumentar a quimio-sensibilidade de células neoplásicas ao tratamento quimioterápico.

Já em um estudo randomizado foi analisado o efeito da suplementação de óleo de peixe em 51 crianças com leucemia com idades entre 4 e 12 anos, resultando que o óleo de peixe tem um efeito positivo sobre o nível de apetite, a ingestão calórica e a musculatura da circunferência do braço. Bem como, benefícios consideráveis à saúde, incluindo entre estes efeitos anti-inflamatórios e antioxidantes (Elbarbary et al., 2015).

Considerando assim, o papel dos ácidos graxos ômega-3 na preservação e restauração da massa muscular em pacientes com câncer, a inclusão da suplementação com óleo de peixe apresenta resultados satisfatórios no ganho ponderal e pode também reduzir a toxicidade associada à quimioterapia. (Laviano et al., 2013). Vale ressaltar, que algumas diretrizes já recomendam a inclusão do ômega 3 durante o tratamento do câncer, com o objetivo de estabilizar a perda de peso (ASPEN, 2009). 
Na pesquisa de Planas et al, (2011) observou que apesar do ômega 3 demonstrar ação anti-inflamatória e antineoplásica os resultados ainda são contestáveis quanto ao uso desse ácido graxo durante a terapia antineoplásica, no entanto, é importante citar que nenhum estudo demostrou efeito deletério ao paciente.

Por fim é interessante descrever que a intervenção nutricional no paciente oncológico ajuda na melhora da qualidade de vida, evitando assim a morte precoce e diminuindo as complicações decorrentes do tratamento. Dessa forma, devendo fazer parte da terapêutica global para esses indivíduos acometidos por tal enfermidade. Esta assistência deverá ser especializada, individualizada e adequada às necessidades do paciente, de acordo com a agressividade e a complexidade do tratamento, utilizando sempre compostos, como exemplo imunomoduladores inclusive o ômega-3, que ajudem de alguma forma na sobrevida do indivíduo com certas neoplasias (SBNPE, 2011).

\section{Considerações finais}

Diante do que foi mencionado no presente estudo uma dieta imunomoduladora com o ômega-3 tem diversos benéficos, sendo um alimento muito importante na dieta dos pacientes com câncer, incluído a leucemia pois age no organismo de várias formas, desempenhando um papel importante nos processos inflamatórios e auxiliando na recuperação de peso e sobrevida do paciente.

\section{Referências}

Associação Brasileira de Linfoma e Leucemia [ABRALE]. 2016. Câncer Infantil - Leucemias. Disponível em: <http://abrale.org.br/leucemia-infantil/o-que-e>. Acesso em: 17 mai. 2019.

Elbarbary, N. S., Ismail, E. A. R., Farahat, R. K., \& El-Hamamsy, M. 2016. $\omega$-3 fatty acids as an adjuvant therapy ameliorates methotrexate-induced hepatotoxicity in children and adolescents with acute lymphoblastic leukemia: A randomized placebo-controlled study. Nutrition, 32(1), 41-47.

Fahrmann, J. F., \& Hardman, W. E. 2013. Omega 3 fatty acids increase the chemo-sensitivity of B-CLL-derived cell lines EHEB and MEC-2 and of B-PLL-derived cell line JVM-2 to anti-cancer drugs doxorubicin, vincristine and fludarabine. Lipids in health and disease, 12(1), 1-13.

Garófolo, A., Lopez, F. A., \& Petrilli, A. S. 2005. High prevalence of malnutrition among patients with solid non-hematological tumors as found by using skinfold and circumference measurements. Sao Paulo Medical Journal, 123, 277-281.

Instituto Nacional De Câncer [INCA]. 2011. Consenso nacional de nutrição oncológica: volume 2. Rio de Janeiro, RJ: INCA.

Instituto Nacional De Câncer [INCA]. 2018. Câncer infanto-juvenil. Disponível em: https://www.inca.gov.br/tipos-de-cancer/cancer-infantojuvenil

Laviano, A., Rianda, S., Molfino, A., \& Fanelli, F. R. 2013. Omega-3 fatty acids in cancer. Current Opinion in Clinical Nutrition \& Metabolic Care, 16(2), 156-161.

Mahan, L. K., \& Escott-Stump, S. 2010. Alimentos nutrição \& dietoterapia. Rio de Janeiro, RJ: Elsevier.

Maruyama, T., Kono, K., Mizukami, Y., Kawaguchi, Y., Mimura, K., Watanabe, M., ... \& Fujii, H. 2010. Distribution of Th17 cells and FoxP3 (+) regulatory T cells in tumor-infiltrating lymphocytes, tumordraining lymph nodes and peripheral blood lymphocytes in patients with gastric cancer. Cancer science, 101(9), 1947-1954.

Mesquita, T. R., Souza, A. A. D., Constantino, E., Pelógia, N. C. C., Posso, I. D. P., \& Pires, O. C. 2011. Antiinflammatory effect of dietary supplementation with omega-3 fatty acids in rats. Revista Dor, 12, 337-341.

Perini, J. Â. D. L., Stevanato, F. B., Sargi, S. C., Visentainer, J. E. L., Dalalio, M. M. D. O., Matshushita, M., ... \& Visentainer, J. V. 2010. Omega-3 and omega-6 polyunsaturated fatty acids: metabolism in mammals and immune response. Revista de Nutrição, 23(6), 1075-1086.

Planas, M., Fernández-Ortega, J. F., \& Abilés, J. 2011. Guidelines for specialized nutritional and metabolic support in the critically-ill patient. Update. Consensus SEMICYUC-SENPE: Oncohematological patient. Nutricion hospitalaria, 26(2), 50-53.

Santos, R. D., Gagliardi, A. C. M., Xavier, H. T., Magnoni, C. D., Cassani, R., Lottenberg, A. M. P., ... \& Ramos, S. 2013. I Diretriz sobre o consumo de gorduras e saúde cardiovascular. Arquivos Brasileiros de Cardiologia, 100(1), 1-40. 
Sociedade Brasileira de Nutrição Parenteral e Enteral [SBNPE]. 2011. Triagem e avaliação do estado nutricional. São Paulo, SP: AMB.

West, N. J., Clark, S. K., Phillips, R. K., Hutchinson, J. M., Leicester, R. J., Belluzzi, A., \& Hull, M. A. 2010. Eicosapentaenoic acid reduces rectal polyp number and size in familial adenomatous polyposis. Gut, 59(7), 918-925.

\section{Minicurrículo}

Jennyfhe Emannuelly da Silva Pereira. Graduanda na Pós Graduação em Nutrição clínica, funcional e estética na Faculdade Integrada de Patos-UNIFIP, Nutricionista graduada no Centro Universitária do Vale Do Ipojuca Wyden, Integrante fundadora da Liga Acadêmica em Nutrição e Saúde Coletiva- LANUSC no UNIFAVIP WYDEN. Foi estagiária em Caruaru nos NASFs, no Hospital Regional do Agreste, e no Hospital Jesus Nazareno (Fusam).

Maria Cecília da Silva. Nutricionista pelo Centro Universitário Vale do Ipojuca / Wyden, (2019). Foi membro da Liga acadêmica em Nutrição e Saúde Coletiva - LANUSC no UNIFAVIP | WYDEN e tem experiência com a disciplina de Avaliação Nutricional Avançada.

Camila Chiara Pereira de Oliveira. Nutricionista e Professora do Centro Universitário UNIFAVIP, Mestre em Nutrição pelo Programa de Pós-Graduação em Nutrição da Universidade Federal de Pernambuco (PPGN UFPE), Especialista em Nutrição Clínica pelo Programa de Residência em Nutrição Clínica do Hospital Universitário Oswaldo Cruz (HUOC - UPE), Especialista em Nutrição Esportiva pela Universidade Gama Filho (UGF). Graduada em Nutrição pela UFPE desde 2009. Tem experiência em Nutrição Clínica, Terapia Nutricional, Paciente Crítico e Alimentação Coletiva.

Como citar: Pereira, J.E.S., Silva, M.C., \& Oliveira, C.C.P., 2021. O impacto do Omega-3 no tratamento da leucemia em crianças e adolescentes. Pubsaúde, 7, a213. DOI: https://dx.doi.org/10.31533/pubsaude7.a213

Recebido: 10 jun. 2021.

Revisado e aceito: 24 jun. 2021.

Conflito de interesse: os autores declaram, em relação aos produtos e companhias descritos nesse artigo, não ter interesses associativos, comerciais, de propriedade ou financeiros que representem conflito de interesse.

Licenciamento: Este artigo é publicado na modalidade Acesso Aberto sob a licença Creative Commons Atribuição 4.0 (CC-BY 4.0). 